\title{
Development of a Simultaneous Analytical Method for Diquat, Paraquat and Chlormequat in Animal Products Using UPLC-MS/MS
}

\author{
Il Kyu $\mathrm{Cho}^{1{ }^{\dagger *}}, \mathrm{Md}$. Musfiqur Rahman ${ }^{1 \dagger}$, Jae Ung Seol ${ }^{1}$, Hyun Ho $\mathrm{Noh}^{2}$, \\ Hyeong-Wook $\mathrm{Jo}^{3}$ and Joon-Kwan Moon ${ }^{4}$
}

${ }^{1}$ Eco-Friendly Agri-Bio Research Center, Jeonnam Bioindustry Foundation, Gokseong 57510, Korea, ${ }^{2}$ Residual Agrochemical Assessment Division, Department of Agro-Food Safety \& Crop Protection, National Institute of Agricultural Sciences, Rural Development Administration, Wanju 55365, Korea, ${ }^{3}$ Hansalim Agro-Food Analysis Center, Hankyong National University Industry Academic Cooperation Foundation, Anseong 17579, Korea, ${ }^{4}$ School of Applied Science in Natural Resources and Environment, Hankyong National University, Anseong 17579, Korea

Received: 3 November 2020/ Revised: 25 November 2020/ Accepted: 30 November 2020

Copyright (C) 2020 The Korean Society of Environmental Agriculture

This is an Open-Access article distributed under the terms of the Creative Commons Attribution Non-Commercial License (http://creativecommons.org/licenses/by-nc/3.0) which permits unrestricted non-commercial use, distribution, and reproduction in any medium, provided the original work is properly cited.

\section{ORCID}

II Kyu Cho

https://orcid.org/0000-0002-4177-5588

Md. Musfiqur Rahman

https://orcid.org/0000-0002-3848-4907

Jae Ung Seol

https://orcid.org/0000-0002-1017-2189

\begin{abstract}
BACKGROUND: The residual analysis of polar pesticides has remained a challenge. It is even more difficult to simultaneously analyze multiple polar pesticides. Diquat, paraquat, and chlormequat are typical examples of highly polar pesticides. The existing methods for the analysis of diquat, paraquat and chlormequat are complex and time consuming. Therefore, a simple, quick and effective method was developed in the represent study for simultaneous analysis of diquat, paraquat and chlormequat in animal
\end{abstract}

\footnotetext{
${ }^{\dagger}$ These authors equally contributed to this paper as first authors

*Corresponding author: Il Kyu Cho

Phone: +82-61-363-9544; Fax: +82-61-362-0631;

E-mail: ilkyucho@naver.com
}

\author{
Hyun Ho Noh \\ https://orcid.org/0000-00027568-8490 \\ Hyeong-Woo Jo \\ https://orcid.org/0000-0002-2271-9767 \\ Joon-Kwan Moon \\ https://orcid.org/0000-0001-9944-7475
}

products, meat and fat using UPLC-MS/MS.

METHODS AND RESULTS: Sample extraction was carried out using acidified acetonitrile and water and reextracted with acidified acetonitrile and combine the extracts followed by centrifugation. The extract was then cleaned up with a HLB cartridge after reconstitution with acidic acetonitrile and water. The method was validated in quintuplicate at three different concentrations. The limits of detection (LOD) and quantification (LOQ) were 0.0015 and $0.005 \mathrm{mg} / \mathrm{L}$, respectively. Matrix suppression effect was observed for all of the analytes. A seven point matrix matched calibration curve was constructed for each of the compound resulted excellent linearity with determination coefficients $\left(R^{2}\right) \geq 0.991$. Accuracy and precision of the method was calculated from the recovery and repeatability and ranged from 62.4 to $119.7 \%$ with relative standard deviation less than $18.8 \%$. 
CONCLUSION: The recovery and repeatability of the developed method were in the acceptable range according to the Codex Alimentarius guideline. The developed method can be applied for the routine monitoring of diquat, paraquat, and chlormequat in animal products, meat and fat.

Key words: Chlormequat, Diquat, Paraquat, Simultaneous Analysis, UPLC-MS/MS

\section{Introduction}

Diquat, paraquat and chlormequat (Fig. 1) are quaternary ammonium, non-selective and nonsystematic contact herbicides. They are widely used for controlling broadleaf, grassy weeds and aquatic weeds [1, 2]. These compounds are commonly known as QUATs and considered as toxic herbicides [1, 3].

Herbicides can enter human body directly or indirectly through food chain. Residues can also be accumulated to the livestock animal via contaminated feed [4]. Various international organizations regulate pesticide residues in foods using maximum residue limits (MRL). According to the Codex guideline, the MRLs of diquat, paraquat and chlormequat were determined $0.005 \mathrm{mg} / \mathrm{kg}$ or above for most of the food products of animal origin. Therefore, a sensitive analytical method is needed for monitoring of these three herbicide in livestock animal products. Several analytical methods have been developed for the analysis of diquat and paraquat in various matrices [2, 3, 5-11]. A few methods were also published for simultaneous analysis of diquat, paraquat and chlormequat using LC-MS/MS [12-16]. Due to high water solubility, these compounds are not included in multi-residue analysis as they required specific types of separation column and mobile phases. However, existing single residue methods are time consuming associated with complex sample preparation involving heating step during sample preparation. The detection of these methods also requires addition of ion pair agent in the mobile phase which demanded continuous cleaning of the instrument after application $[6,7,10-12,14$, 15].

To overcome such problems, QuPPE (Quick Polar Pesticides) method has been introduced and used for the analysis of polar pesticides such as QUATs in various matrices $[8,17,18]$. The method involved extraction with methanol and acid aqueous solution resulted a high amount of matrix co-extractives and the measurement was carried out using isotope labeled internal standard that make the experiment expensive and difficult.

Therefore, the aim of the present study was to develop a simple and effective method for the analysis of diquat, paraquat, and chlormequat in livestock animal products using UPLC-MS/MS.

\section{Materials and Methods}

\section{Chemicals and Reagents}

Standard diquat and paraquat calibration mixture $(1000 \mathrm{mg} / \mathrm{L})$ in water was purchased from Restek (Bellefonte, PA, USA). Chlormequat standard (purity 98.2\%) was supplied by Sigma Aldrich (St. Louis, MO, USA). HPLC grade acetonitrile and water were purchased from Honeywell (Muskegon, MI, USA). Analytical grade formic acid (purity $\geq 95 \%$ ) and ammonium format (purity $\geq 99.995 \%$ ) were obtained from Sigma Aldrich (St. Louis, MO, USA).

\section{Preparation of standard solution and calibration}

An amount of $10.18 \mathrm{mg}$ of chlormequat was dissolve in $10 \mathrm{~mL}$ water to prepare $1000 \mathrm{ppm}$ chlormequat. The mixed diquat and paraquat solution was added with the standard chlormequat to provide the mixed solution at $100 \mathrm{ppm}$ of each. The intermediate standard solution was prepared by diluting the standard solution to $10 \mathrm{ppm}$ and $1.0 \mathrm{ppm}$ with mobile phase B. The working standard solutions were prepared by diluting the intermediate

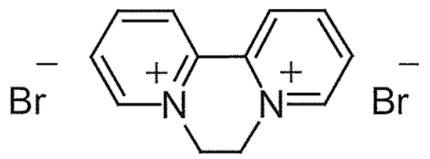

(a)

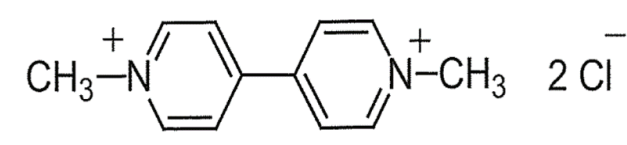

(b)

$$
\mathrm{ClCH}_{2} \mathrm{CH}_{2} \stackrel{+}{\mathrm{N}}\left(\mathrm{CH}_{3}\right)_{3} \mathrm{Cl}
$$

(c)

Fig. 1. Chemical structures of diquat dibromide (a), paraquat (b) and chlormequat (c). 
standard solution to $0.001,0.002,0.004,0.006,0.008,0.01$, and $0.012 \mathrm{mg} / \mathrm{L}$ with the same solvent.

\section{Sample Preparation}

An each $5 \mathrm{~g}$ of homogenized livestock animal products (chicken, pork, pork fat, beef, beef fat, egg and milk) was taken in a $50 \mathrm{~mL}$ Teflon centrifuge tube to which a $15 \mathrm{~mL}$ of $0.5 \%$ formic acid in acetonitrile and $5 \mathrm{~mL}$ of $0.5 \%$ formic acid in water was added and extract with a Geno Grinder (SPEX Sample Prep., USA) for 2 $\mathrm{min}$ at $1200 \mathrm{rpm}$. The extracts were then centrifuged at $3500 \mathrm{rpm}$ for $5 \mathrm{~min}$. and transferred the whole solution to another centrifuge tube. The remaining samples were re-extracted with $10 \mathrm{~mL}$ of $0.5 \%$ formic acid in acetonitrile and transfer and combine the solution to the centrifuge tube following centrifugation. The mixed solutions were vortexed for $10 \mathrm{~s}$ and evaporated the organic layer through a gentle $\mathrm{N}_{2}$ stream at $40^{\circ} \mathrm{C}$. The remaining aqueous solution was adjusted to reconstructing until $6.25 \mathrm{~mL}$ with $0.5 \%$ formic acid in water and additional $6.25 \mathrm{~mL}$ of $0.5 \%$ formic acid in acetonitrile was added. The total $12.5 \mathrm{~mL}$ of mixed solution was vortexed for 10 sec. prior to purification.

An aliquot of $2 \mathrm{~mL}$ of extract was loaded to a HLB (500 mg) LP cartridges (Oasis, Waters, Milford, MA, USA) that was previously conditioned with $6 \mathrm{~mL}$ of $0.5 \%$ formic acid in water and $6 \mathrm{~mL}$ of $0.5 \%$ formic acid in acetonitrile with an solid phase extraction (SFE) manifold under a reduced pressure. The loaded extract was then eluted with a $1.6 \mathrm{~mL}$ of $0.5 \%$ formic acid in acetonitrile. The eluents were combined in a $15 \mathrm{~mL}$ Teflon tube and added with $0.4 \mathrm{~mL}$ of $0.5 \%$ formic acid in acetonitrile followed by vortexing. One $\mathrm{mL}$ was transferred to a 2 $\mathrm{mL}$ plastic vial followed by addition of $10 \mu \mathrm{L}$ of 5000 $\mathrm{mM}$ ammonium formate.

\section{Method validation}

The method was validated according to the Codex Alimentarius guidelines in term of limits of detection (LOD), limits of quantification (LOQ), linearity, matrix effect selectivity, specificity, accuracy and precision. LOD was the lowest detectable amount of analyte that produced signal three times higher than the background noise. The lowest quantifiable amount of analyte was termed as LOQ that generated a signal ten times higher than the base line noise. Linearity is determined from the co-efficient of determinants $\left(R^{2}\right)$ obtained from the seven points calibration curve. Matrix effect was compensated by constructing matrix matched calibration and the effect was calculated by comparing the slope of the matrix matched calibration and the slope of the matrix free solvent calibration. Accuracy was determined from the recovery of fortified standard following sample preparation procedure and according to the guideline recovery must be within the range of $50 \sim 120 \%$ depending on the spiking concentration. Precision was the outcome of five times repeatability of the recovery experiments and was expressed as a relative standard deviation, RSD. According to the Codex guideline the valid range for precision is RSD $<22 \sim 32 \%$ of the spiking concentrations.

\section{Instrument}

An ultra-performance liquid chromatograph (Waters AQUITY UHPLC H Class, Waters, Hertfordshire, UK) coupled with a mass spectrometer (AB SCIEX QTRAP 3500 mass spectrometer, SCIEX, Redwood, CA, USA) was employed for detection and quantification of diquat, paraquat, and chlormequat. The MS/MS was carried out in multi-reaction monitoring mode (MRM) with positive electron spray ionization. For MRM method development a syringe pump was equipped with the MS/MS system for standard infusion and optimization of compound ionization. The chromatographic separation was carried out on a Hilic-Si column $(50 \times 2.1 \mathrm{~mm}, 2.7 \mu \mathrm{m})$. The column oven temperature was maintained at $45^{\circ} \mathrm{C}$. A binary solvent system composed of $50 \mathrm{mM}$ ammonium formate and $0.5 \%$ formic acid in water (A), and $50 \mathrm{mM}$ ammonium formate and $0.5 \%$ formic acid in $75 \%$ ACN and $25 \%$ water (B). The gradient mobile phase started with $100 \%$ B which was reduced to $65 \%$ B at $4 \mathrm{~min}$. At $4.1 \mathrm{~min}$ it was suddenly increased to $100 \% \mathrm{~B}$ and remains constant until $7 \mathrm{~min}$. The column flow was maintained at $0.60 \mathrm{~mL} / \mathrm{min}$ with the injection volume of $10 \mu \mathrm{L}$. The ion source temperature was $500^{\circ} \mathrm{C}$ and the spray voltage was $5500 \mathrm{~V}$. Nitrogen was used as nebulizer, curtain, and heating gas. The optimized MS/MS parameter is shown in Table 1.

\section{Results and Discussion}

The extraction method was optimized based on the physicochemical properties of diquat, paraquat, and chlormequat. The negative octanol-water co-efficient of these three compounds indicated that the translocation of these three analytes from aqueous phase to organic phase is almost impossible. Therefore, the most popular 
Table 1. Multiple reaction monitoring (MRM) conditions for diquat, paraquat and chlormequat

\begin{tabular}{cccccccc}
\hline Compound & $\begin{array}{c}\text { Precusor ion } \\
\mathrm{m} / \mathrm{z}\end{array}$ & $\begin{array}{c}\text { Product ion } \\
\mathrm{m} / \mathrm{z}\end{array}$ & $\begin{array}{c}\mathrm{RT} \\
(\mathrm{m})\end{array}$ & $\begin{array}{c}\mathrm{DP} \mathrm{P}^{\mathrm{a})} \\
(\mathrm{v})\end{array}$ & $\begin{array}{c}\mathrm{EP}^{\mathrm{b})} \\
(\mathrm{v})\end{array}$ & $\begin{array}{c}\mathrm{CE}^{\mathrm{c})} \\
(\mathrm{v})\end{array}$ & $\begin{array}{c}\mathrm{CXP}^{\mathrm{d})} \\
(\mathrm{v})\end{array}$ \\
\hline \multirow{2}{*}{ Diquat } & 183.300 & 130.1 & 3.79 & 100 & 10 & 50 & 12 \\
& 183.300 & 157.2 & 3.79 & 150 & 10 & 35 & 3 \\
\hline \multirow{2}{*}{ Paraquat } & 171.300 & 77.2 & 4.14 & 100 & 10 & 43 & 14 \\
& 185.300 & 170.3 & 4.14 & 150 & 10 & 23 & 6 \\
\hline \multirow{2}{*}{ Chlormequat } & 122.652 & 59.1 & 0.57 & 81 & 10 & 47 & 6 \\
& 122.652 & 58.6 & 0.57 & 81 & 10 & 47 & 6 \\
\hline
\end{tabular}

${ }^{\mathrm{a})}$ Declustering potential, ${ }^{\mathrm{b})}$ Enterance potential energy, ${ }^{\mathrm{c})}$ Collision energy, ${ }^{\mathrm{d})}$ Collision cell exit potential

sample preparation method like QuEChERS (Quick, Easy, Cheap, Effective, Rugged, and Safe) is not applicable as the method involved salting out for transfer of target analyte to the organic phase. Since all these three compounds are stable in an acidic environment, formic acid was therefore used with acetonitrile and water for extraction, purification and detection. During MS/MS analysis it was observed that if the compounds are dissolved in mobile phase B (0.5\% formic acid in 75\% $\mathrm{ACN}$ and 25\% water), a good separation and sensitivity of these three compounds can be then achieved. Since extraction and purification procedure were subjected with water, it was difficult to evaporate and reconstitute the purified extract in mobile phase B. Therefore, extraction and purification steps were designed in such a way so that the final purified extract was exactly the same as the mobile B. To do so, during extraction, acidic acetonitrile and water fraction was maintained a fifty-fifty ratio; after complete evaporation of organic phase, it turned to 75:25 $\left(\mathrm{ACN}: \mathrm{H}_{2} \mathrm{O}\right)$ after cartridge purification.

During optimization of HLB cartridge cleanup conditions, none of the compounds were retained in the cartridge after loading. After a $2.0 \mathrm{~mL}$ of extract containing $0.5 \%$ formic acid in fifty-fifty $\left(\mathrm{ACN}: \mathrm{H}_{2} \mathrm{O}\right)$ was loaded on a HLB cartridge, elution was performed with $0.5 \% \mathrm{FA}$ in $1.6 \mathrm{~mL} \mathrm{ACN}$. Finally, the volume was adjusted to $4 \mathrm{~mL}$ with the addition of $0.4 \mathrm{~mL}$ of $0.5 \%$ formic acid in ACN. This volume adjustment was utilized for the matrix matched calibration. From this $4 \mathrm{~mL}$ purified extract, $1 \mathrm{~mL}$ was transferred to a $2 \mathrm{~mL}$ plastic vial flowed by addition of $10 \mu \mathrm{L}$ of $5000 \mathrm{mM}$ ammonium formate for exact matching of mobile phase B. In the whole extraction, purification and detection process, no glassware were used due to the glass adhesive properties of these compounds [17].

The LOD and LOQ of the method for the three compounds were 0.0015 and $0.005 \mathrm{mg} / \mathrm{kg}$ respectively.
The LOQ of the established method was either at MRL (maximum residue limit) level or below the MRL regulated by Codex Alimentarius guidelines for animal food products.

Fig. 2 shows the total ion chromatograms of matrix matched standard mixture, blank, and recovery. Similar retention time between standard and recovery, and absence (or less than $30 \%$ of LOQ) of interferences at the blank represents the selectivity and specificity of the method.

Extensive matrix suppression effect for all of the three compounds was observed ( -25.37 to $-80.34 \%)$ even after cartridge purification and fivefold dilution. It might be due to the addition of high amount of buffering salt (50 $\mathrm{mM}$ ammonium format) required for optimum column efficiency. Therefore, a seven point $(0.005,0.01,0.02,0.03$, $0.04,0.05,0.06 \mathrm{mg} / \mathrm{kg}$ ) matrix matched calibration curve was constructed for all of the compounds in each of the matrices that provided good linearity with determination co-efficient $\left(R^{2}\right) \geq 0.9912$. The recovery and repeatability of the present methods were ranging $62.4 \sim 119.7 \%$ and RSD $\leq 18.8 \%$ respectively in all of the matrices. The linearity, matrix effect, recovery and repeatability of these three QUATs are shown in Table 2. In the case of paraquat, below $70 \%$ recovery was observed at LOQ level and 2LOQ level (only in egg matrices) indicated that low concentration of paraquat is bit unstable. Even though recovery of paraquat was below $70 \%$, it was still within the acceptable range according to the Codex Alimentarius (60 120\%) (CAC/GL 90-2017). Likely, the matrix suppression effect showed maximum over $80 \%$. The $20 \%$ of matrix effect recognized by the Ministry of Food and Drug Safety is a standard guideline for whether matrix matched should be performed or not, and the percentage of matrix effect is meaningless if matrix matched is conducted. Diquat, paraquat and chlormequats are cationic compounds that ion suppress 

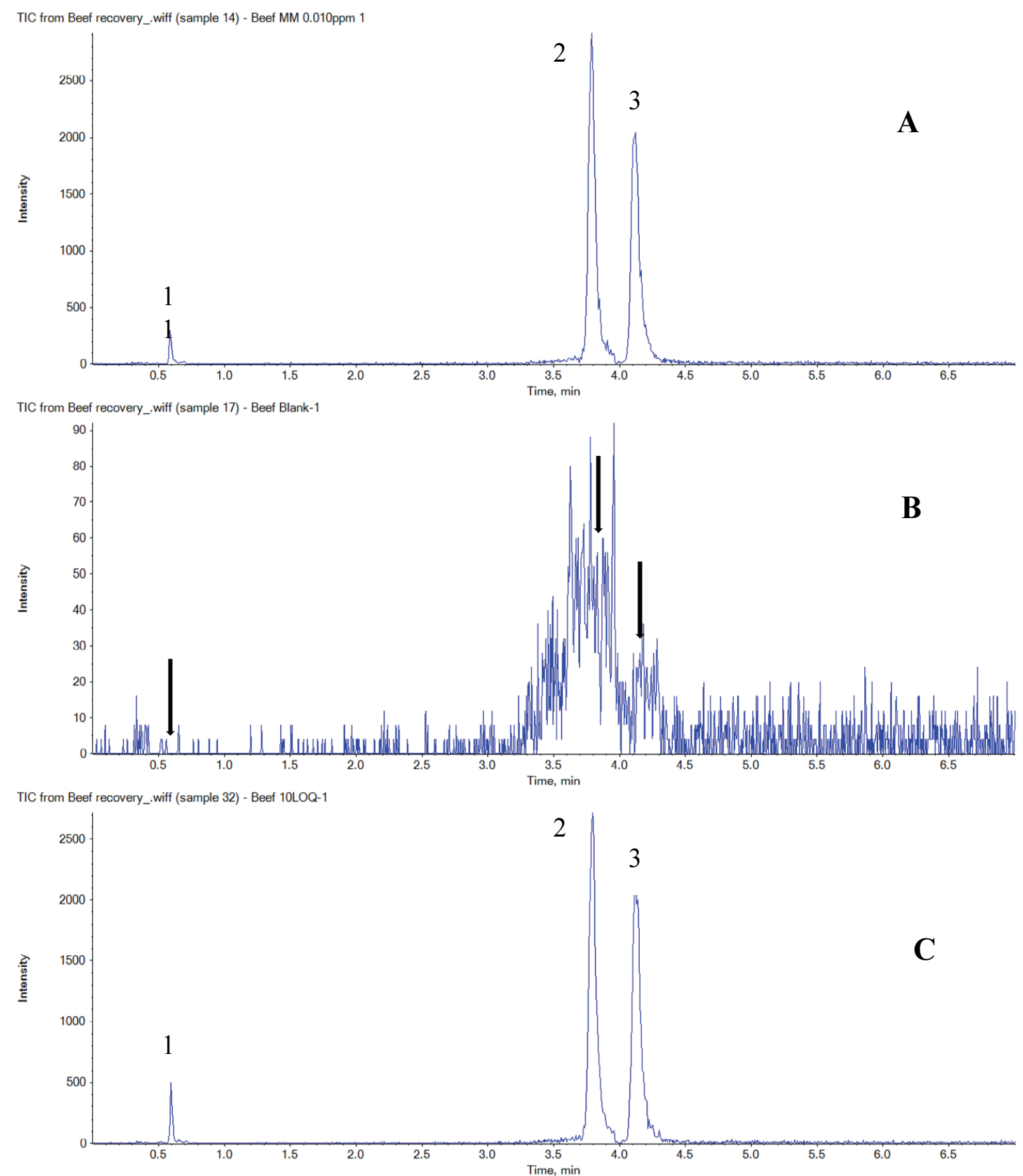

Fig. 2. UPLC-MS/MS TIC chromatograms of matrix matched standard (10 LOQ level) mixture of diquat (A), blank beef matrix (B), recovery at $10 \mathrm{LOQ}$ level (C) (a; chlormequat, b; diquat dibromide, c; paraquat). The numbers are as 1: chlormequat; 2: diquat dibromide; 3: paraquat. The arrow symbols in the panel $\mathrm{B}$ represent the retention times of chlormequat, diquat dibromide and paraquat, respectively.

takes a place by using solid phase extraction method with ion exchange resin other than HLB cartridge. While SPE SCX (Strong Cation Exchange) cartridge was used to utilize strong cation exchange chromatography, binding of compounds in the SPE occur but them did not eluted from the cartridge. An ion enhancement or ion suppression occurred when SPE purification with dispersive SPE and Envi-carb was carried to purify the compounds from the animal products. It was same as the elution solution of the compounds was passed through a syringe filter 
Table 2. Linearity, matrix effect and recovery of diquat, paraquat and chlormequat livestock animal products

\begin{tabular}{|c|c|c|c|c|c|c|}
\hline \multirow{2}{*}{ Matrix } & \multirow{2}{*}{ Pesticides } & \multirow{2}{*}{$\begin{array}{c}\text { Linearity } \\
\left(R^{2}\right)\end{array}$} & \multirow{2}{*}{ Matrix effect } & \multicolumn{3}{|c|}{ Recovery \pm RSD $^{\text {a) }}(\%)$} \\
\hline & & & & $1 \mathrm{LOQ}$ & 2 LOQ & $10 \mathrm{LOQ}$ \\
\hline \multirow{3}{*}{ Chicken } & Diquat & 0.9973 & -25.371 & $81.0 \pm 7.3$ & $80.6 \pm 8.06$ & $90.5 \pm 9.9$ \\
\hline & Paraquat & 0.9949 & -25.641 & $64.7 \pm 3.8$ & $78.0 \pm 9.5$ & $99.5 \pm 6.3$ \\
\hline & Chlormequat & 0.9922 & -80.079 & $90.2 \pm 19.6$ & $99.6 \pm 10.3$ & $107.9 \pm 3.9$ \\
\hline \multirow{3}{*}{ Pork } & Diquat & 0.9986 & -33.665 & $89.2 \pm 6.0$ & $92.5 \pm 9.0$ & $92.5 \pm 4.6$ \\
\hline & Paraquat & 0.9981 & -40.773 & $71.2 \pm 7.7$ & $83.8 \pm 5.6$ & $96.1 \pm 5.6$ \\
\hline & Chlormequat & 0.9938 & -80.338 & $107.0 \pm 14.2$ & $116.3 \pm 10.6$ & $111.6 \pm 3.7$ \\
\hline \multirow{3}{*}{ Pork fat } & Diquat & 0.9980 & -29.470 & $87.7 \pm 7.0$ & $88.1 \pm 9.2$ & $94.4+9.3$ \\
\hline & Paraquat & 0.9968 & -37.472 & $65.2 \pm 7.3$ & $80.9 \pm 9.3$ & $102.1 \pm 6.8$ \\
\hline & Chlormequat & 0.9973 & -78.126 & $118.4 \pm 9.6$ & $106.2 \pm 7.4$ & $109.2 \pm 4.6$ \\
\hline \multirow{3}{*}{ Beef } & Diquat & 0.9964 & -40.935 & $84.9 \pm 11.8$ & $85.9 \pm 6.2$ & $85.4 \pm 8.1$ \\
\hline & Paraquat & 0.9966 & -60.380 & $67.2 \pm 5.0$ & $76.9 \pm 9.3$ & $98.2 \pm 4.2$ \\
\hline & Chlormequat & 0.9935 & -87.923 & $99.4 \pm 17.3$ & $111.6 \pm 18.8$ & $118.9 \pm 4.3$ \\
\hline \multirow{3}{*}{ Beef fat } & Diquat & 0.9968 & -43.472 & $84.1 \pm 9.4$ & $92.8 \pm 9.9$ & $83.7 \pm 5.6$ \\
\hline & Paraquat & 0.9993 & -57.767 & $68.7 \pm 5.6$ & $81.2 \pm 7.2$ & $93.1 \pm 7.4$ \\
\hline & Chlormequat & 0.9912 & -87.356 & $119.7 \pm 11.0$ & $107.1 \pm 10.5$ & $106.7 \pm 9.2$ \\
\hline \multirow{3}{*}{ Egg } & Diquat & 0.9920 & -36.528 & $79.3 \pm 7.8$ & $79.3 \pm 5.6$ & $84.9 \pm 4.9$ \\
\hline & Paraquat & 0.9933 & -49.098 & $62.4 \pm 9.5$ & $67.0 \pm 4.8$ & $87.0 \pm 2.3$ \\
\hline & Chlormequat & 0.9991 & -73.546 & $116.3 \pm 11.7$ & $99.3 \pm 10.9$ & $90.1 \pm 8.3$ \\
\hline \multirow{3}{*}{ Milk } & Diquat & 0.9930 & -31.865 & $86.4 \pm 8.1$ & $86.1 \pm 9.3$ & $86.7 \pm 4.6$ \\
\hline & Paraquat & 0.9954 & -25.972 & $64.8 \pm 4.8$ & $73.5 \pm 6.2$ & $79.2 \pm 5.0$ \\
\hline & Chlormequat & 0.9988 & -73.788 & $102.6 \pm 8.8$ & $94.1 \pm 5.8$ & $91.3 \pm 3.7$ \\
\hline
\end{tabular}

${ }^{\text {a) }}$ Mean values of 5 time repetitions with relative standard deviation

An analytical method for diquat and paraquat have been developed involving extraction with a mixture of methanol/water/hydrochloric acid and heating at $80^{\circ} \mathrm{C}$ (Thomas, 2017). The method provided recoveries of 92 120\% in potato, cereals and pulses. However, the LOQ of the method was set at 0.05 or $0.1 \mathrm{mg} / \mathrm{kg}$.

[18] published a method for QUATs in food of plant origin involving acidified mixture of methanol-water for extraction resulted $64 \sim 97 \%$ recovery with the LOQ of $0.01 \mathrm{mg} / \mathrm{kg}$.

[2] determined diquat and paraquat in potato using acidic methanol and a heating step during extraction. Although method provided good recovery (87 106\%), it still required time consuming heating step. Chlormequat was not included in that study.

[8] tried to recover QUATs in olive oil and olive using a QuPPE method. Poor recoveries were observed for diquat and paraquat. Therefore, the developed method that satisfies all of the parameters of international standard can be used for regular monitoring of QUATs in livestock animal products

\section{Note}

The authors declare no conflict of interest.

\section{Acknowledgment}

This work was supported by the National Institute of Food and Drug Safety Evaluation (Project No.: 19162556).

\section{References}

1. Raina-Fulton R (2014) A review of methods for the analysis of orphan and difficult pesticides: glyphosate, glufosinate, quaternary ammonium and phenoxy acid herbicides, and dithiocarbamate and phthalimide fungicides. Journal of AOAC International, 97, 965-977. https:// doi.org/10.5740/jaoacint.SGERaina-Fulton. 
2. Chamkasem N, Harmon TM (2017) Determination of paraquat and diquat in potato by liquid chromatography/ tandem mass spectrometer. Journal of Regulatory Science, 5, 1-8.

https: / / doi.org/10.13140/RG.2.2.28928.02564.

3. Nagatomi Y, Yoshioka T, Yanagisawa M, Uyama A, Mochizuki N (2013) Rapid analysis of paraquat and diquat in beer and malt using LC-MS/MS. Journal of Pesticide Science, 38(3), 144-146. https: / / doi.org/10.1584/jpestics.D13-008.

4. Panseri S, Biondi PA, Vigo D, Communod R, Chiesa LM (2013) Occurrence of organochlorine pesticides residues in animal feed and fatty bovine tissue. Food Industry, 13, 261-283. https://doi.org/10.5772/54182.

5. Kolberg DIS, Mack D, Anastassiades M, Hetmanski MT, Fussell RJ, Meijer T, Mol HGJ (2012) Development and independent laboratory validation of a simple method for the determination of paraquat and diquat in potato, cereals and pulses. Analytical and Bioanalytical Chemistry, 404, 2465-2474.

https: / / doi.org/10.1007/ s00216-012-6340-9.

6. Taguchi VY, Jenkins SWD, Crozier PW, Wang DT (1998) Determination of diquat and paraquat in water by liquid chromatography- (electrospray ionization) mass spectrometry. Journal of the American Society for Mass Spectrometry, 9, 830-839.

https: / / doi.org/10.1016/S1044-0305(98)00043-9.

7. Aramendía MA, Borau V, Lafont F, Marinas A, Marinas JM, Moreno JM, Porras JM, Urbano FJ (2006) Determination of diquat and paraquat in olive oil by ion-pair liquid chromatography-electrospray ionization mass spectrometry (MRM). Food Chemistry, 97, 181-188. https: / / doi.org/10.1016/j.foodchem.2005.05.005.

8. Nortes-Méndez R, Robles-Molina J, López-Blanco R, Vass A, Molina-Díaz A, Garcia-Reyes JF (2016) Determination of polar pesticides in olive oil and olives by hydrophilic interaction liquid chromatography coupled to tandem mass spectrometry and high resolution mass spectrometry. Talanta, 158, 222-228.

https: / / doi.org/10.1016/j.talanta.2016.05.058.

9. Pateiro-Moure M, Martínez-Carballo E, Arias-Estévez M, Simal-Gándara J (2008) Determination of quaternary ammonium herbicides in soils Comparison of digestion, shaking and microwave-assisted extractions. Journal of Chromatography A, 1196-1197, 110-116.

https: / / doi.org/10.1016/j.chroma.2008.03.081.

10. Ariffin MM, Anderson RA (2006) LC/MS/MS analysis of quaternary ammonium drugs and herbicides in whole blood. Journal of Chromatography B, 842, 91-97. https: / / doi.org/10.1016/j.jchromb.2006.03.051.

11. Wang K-C, Chen S-M, Hsu J-F, Cheng S-G, Lee C-K (2008) Simultaneous detection and quantitation of highly water-soluble herbicides in serum using ionpair liquid chromatography-tandem mass spectrometry. Journal of Chromatography B, 876, 211-218. https: / / doi.org/10.1016/j.jchromb.2008.10.042.

12. Castro R, Moyano E, Galceran MT (2001) Determination of quaternary ammonium pesticides by liquid chromatography-electrospray tandem mass spectrometry. Journal of Chromatography A, 914, 111-121. https: / / doi.org/10.1016/S0021-9673(01)00523-4.

13. Nardin T, Barnaba C, Abballe F, Trenti G, Malacarne M, Larcher R (2017) Fast analysis of quaternary ammonium pesticides in food and beverages using cation-exchange chromatography coupled with isotope-dilution highresolution mass spectrometry. Journal of Separation Science, 40, 3928-3937. https: / / doi.org/10.1002/jssc.201700579.

14. Castro R, Moyano E, Galceran MT (2000) On-line ionpair solid-phase extraction-liquid chromatography-mass spectrometry for the analysis of quaternary ammonium herbicides. Journal of Chromatography A, 869, 441-449. https: / / doi.org/10.1016/S0021-9673 (99)01065-1.

15. Vidal JLM, Vega AB, López FJS, Frenich AG (2004) Application of internal quality control to the analysis of quaternary ammonium compounds in surface and ground water from Andalusia (Spain) by liquid chromatography with mass spectrometry. Journal of Chromatography A, 1050, 179-184.

https: / / doi.org/10.1016/j.chroma.2004.08.023.

16. Danezis GP, Anagnostopoulos CJ, Liapis K, Koupparis MA (2016) Multi-residue analysis of pesticides, plant hormones, veterinary drugs and mycotoxins using HILIC chromatography - MS/MS in various food matrices. Analytica Chimica Acta, 942, 121-138. https: / / doi.org/10.1016/j.aca.2016.09.011.

17. Anastassiades M, Kolberg DI, Eichhorn E, Benkenstein A, Lukačević S, Mack D, Wildgrube C, Barth A, Sigalov I et al. (2015) Quick method for the analysis of numerous highly polar pesticides in foods of plant origin via LC-MS/MS involving simultaneous extraction with methanol (QuPPe-method). EU Reference Laboratories for Residues of Pesticides, 9, 1-60.

18. Kaczy'nski P (2017) Clean-up and matrix effect in LC -MS/MS analysis of food of plant origin for high polar herbicides. Food Chemistry, 230, 524-531. https: / / doi.org/10.1016/j.foodchem.2017.03.091. 